\title{
Correspondence
}

\section{Lack of FADD in Tie-2 expressing cells causes RIPK3-mediated embryonic lethality}

\author{
Cunxian Fan ${ }^{1,2}$, Wenjuan $\mathrm{Pu}^{1,2}$, Xiaoxia Wu ${ }^{1}$, Xixi Zhang ${ }^{1}$, Lingjuan $\mathrm{He}^{1}$, Bin $\mathrm{Zhou}^{\star, 1}$ and Haibing Zhang ${ }^{\star, 1}$
}

Cell Death and Disease (2016) 7, e2351; doi:10.1038/cddis.2016.251; published online 1 September 2016

\section{Dear Editor,}

Programmed cell death has an essential role in development and homeostasis of mammalians. Fas-associated death domain (FADD) interacts with the death domain of receptors, leading to the activation of caspase-8, which subsequently activates several downstream caspases and finally executes apoptosis. ${ }^{1}$ Ablation of Caspase-8 or Fadd resulted in embryonic lethality at around E10.5, which implicates a non-apoptosis function of these proteins in embryonic development. $^{2}$ Recently, extensively genetic studies have shown that embryonic lethality caused by Fadd (or Caspase-8) deletion can be rescued by Ripk3 or Ripk 1 ablation. ${ }^{2}$ However, it remains unclear which targeted cell type is responsible for the lethality of $\mathrm{Fadd}^{-1}$ mice.

Mice with conditional deletion of Fadd in immune cells, skin or intestine produced no lethality. ${ }^{2}$ Given the fact that mice with Caspase-8 deficiency in endothelium, employing Tie1-cre promotor, resemble the embryonic lethality of Caspase-8 germline knockout associated with cardiac defects, ${ }^{3}$ we hypothesized that embryonic lethality of Fadd knockout might also attribute to the loss of Fadd in endothelial cells. To directly test this hypothesis, we took advantage of the mice that expressed a functional FADD:GFP fusion gene to reconstitute $\mathrm{Fadd}^{-1}$ mice, and generated tissue-specific Fadd deletion mice using cre-recombinase under the control of tissuespecific promoter, which were reported previously. ${ }^{4}$

First, we specifically deleted FADD:GFP in cardiomyocytes and cardiac progenitor cells by crossing the mice $\left(\mathrm{Fadd}^{-1-} \mathrm{Fadd}_{\mathrm{gfp}}{ }^{+}\right.$) individually with transgenic mice expressing the cTnt-cre and Nkx2.5-cre. cTnt-Cre efficiently delete target genes in myocardium and Nkx2.5-cre targets cardiomyocyte progenitors. ${ }^{5,6}$ We found that both Fadd $^{-1}$ Fadd: $^{2}$ gfp $^{+}$CTnt-Cre $^{+}$and Fadd ${ }^{-1}$ Fadd:gfp ${ }^{+} \mathrm{Nk} 2.5-\mathrm{Cre}^{+}$mice develop normally at E11.5 (Figure 1a). These data indicate that it is loss of Fadd in other types of cells causing embryonic death of $\mathrm{Fadd}^{-/}$mice, not cardiomyocytes or cardiac progenitor cells. We then generated Fadd deficiency in Tie-2 expressing cells by crossing Fadd ${ }^{-1} \mathrm{Fadd}_{\text {:gfp }}{ }^{+}$mice with a transgenic line that expresses cre-recombinase under the control of Tie-2 promoter. In contrast, Fadd ${ }^{-1}$ Fadd:gfp $^{+}$Tie2- $^{-}$
$\mathrm{Cre}^{+}$mice died at E11.5 with the same cardiovascular defects as $\mathrm{Fadd}^{-1}$ mice, such as vessel defect and pericardial bleeding, suggesting that hemodynamic failure resulting in embryonic death could be owing to abnormal cardiovascular development (Figure 1a). Whole-mount staining for endothelial cell marker PECAM and FADD surrogates GFP showed that FADD:GFP was expressed in endothelial cells in $\mathrm{Fadd}^{-/}$ Fadd:gfp ${ }^{+}$mice (yellow). However, FADD:GFP was not detected in endothelial cells of Fadd ${ }^{+-} \mathrm{Fadd}_{\text {:gfp }} \mathrm{Tie}^{+}-\mathrm{Cre}^{+}$ embryo (Figure 1b), whereas FADD:GFP was still expressed in non-endothelial cells such as cardiomyocytes (Figure 1b). These data indicate that Tie2-cre efficiently ablates FADD: GFP in endothelial cells. Compared with normal embryos, Fadd $^{-1}$ Fadd:gfp $^{+}$Tie2-Cre $^{+}$displayed a low degree of trabeculation in the walls of the common ventricular chamber and endocardial cushion defect by reduced endothelial-tomesenchymal formation (Figure 1c), suggesting that loss of Fadd in endothelial cells causes endocardium-related cardiac development defect. Although the lethality of $\mathrm{Fadd}^{-1} \mathrm{Fadd}$ $\mathrm{gfp}^{+} \mathrm{Tie2}-\mathrm{Cre}^{+}$is caused by the same pathology as $\mathrm{Fadd}^{-1}$ embryo at E11.5, ${ }^{7,8}$ we asked whether this lethality of $\mathrm{Fadd}^{-1}-$ Fadd:gfp ${ }^{+}$Tie2-cre $^{+}$is mediated by RIPK3 as Fadd $^{-1}$ mice. Therefore, we crossed Ripk3 knockout allele to the Fadd $^{-/}$ Fadd:gfp ${ }^{+}$Tie2-Cre mice and found that embryonic lethality of Fadd $^{-1}{ }^{-}$Fadd:gfp $^{+}$Tie2-Cre $^{+}$mice at E11.5 was rescued by Ripk3 deletion. Furthermore, Fadd ${ }^{-1}$ Fadd:gfp $^{+}$Tie2- $^{-}$ $\mathrm{Cre}^{+}$Ripk $^{-/-}$embryos displayed normal degree of trabeculation in the walls of the common ventricular chamber and normal cushion development as Fadd $^{-1}{ }^{-}$Fadd:gfp ${ }^{+}$embryos (Figure 1c). Given that Tie2 is predominantly expressed in endothelial cells and hematopoietic cells, embryonic lethality of Fadd ${ }^{-1}$ Fadd:gfp ${ }^{+}$Tie2-Cre ${ }^{+}$mice at E11.5 might be owing to Fadd deletion in both endothelial and hematopoietic populations. In addition, hematopoietic stem cells are mainly derived from aortic endothelial cells during early embryonic development, and the role of FADD in hematopoietic development could also be secondary to the defect in endothelial cells. More specific genetic tools that could distinguish hematopoietic cells and endothelial cells are needed to dissect the roles of FADD in these two populations. Taken together, these results

\footnotetext{
${ }^{1}$ Key Laboratory of Nutrition and Metabolism, Institute for Nutritional Sciences, Shanghai Institutes for Biological Sciences, Chinese Academy of Sciences, University of Chinese Academy of Sciences, Shanghai 200031, China

*Corresponding author: B Zhou or H Zhang, Key Laboratory of Nutrition and Metabolism, Institute for Nutritional Sciences, Shanghai Institutes for Biological Sciences, University of Chinese Academy of Sciences, 294 Taiyuan Road, Shanghai 200031, China. Tel: +86 215492 0988; Fax: +86 21 5492 0988; E-mail: hbzhang@ sibs.ac.cn or zhoubin@sibs.ac.cn

${ }^{2}$ These authors contributed equally to this work.
} 
a

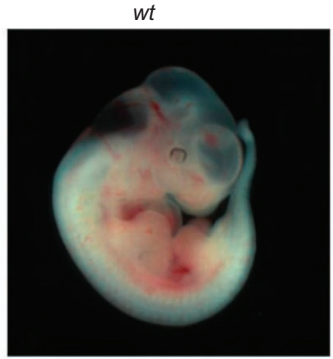

Fadd ${ }^{-}$Fadd:gfp ${ }^{+}$

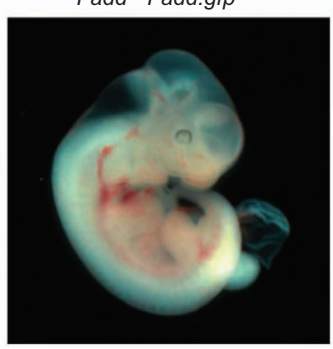

Fadd ${ }^{-}$Fadd:gfp ${ }^{+}$Tie2-Cre ${ }^{+}$

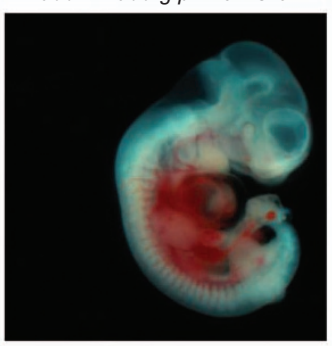

b
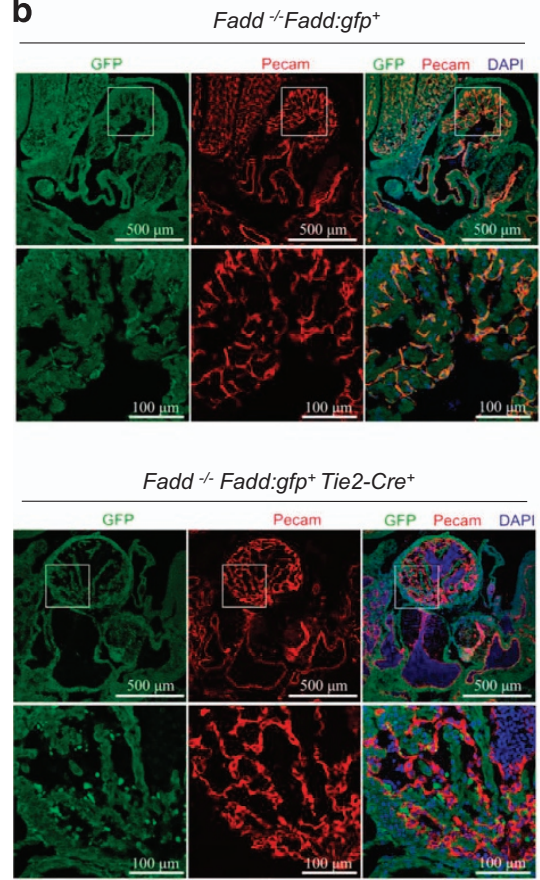

Fadd $\%$

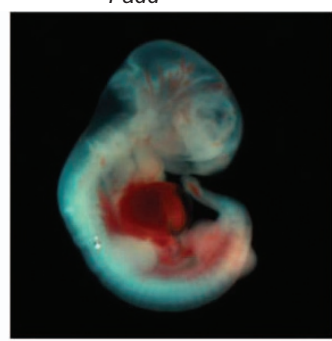

Fadd ${ }^{-}$Fadd:gfp ${ }^{+}$cTnt-Cre ${ }^{+}$

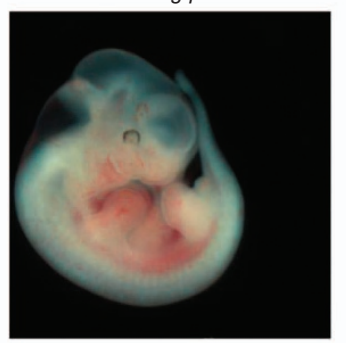

Fadd ${ }^{-}$Fadd:gfp ${ }^{+}$Tie2-Cre ${ }^{+}$Ripk3\% ${ }^{--}$

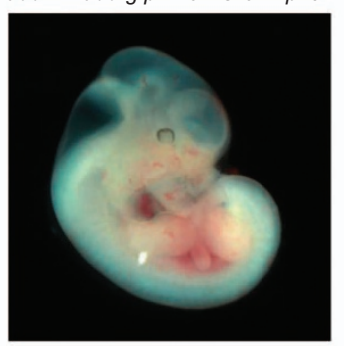

Fadd $\%$ Fadd:Gfp ${ }^{+}$Nkx2.5-Cre ${ }^{+}$

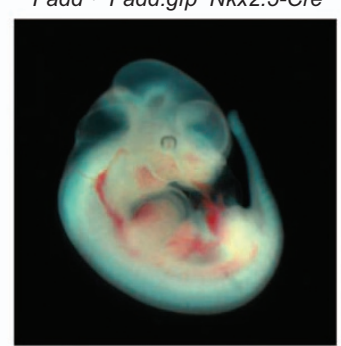


c

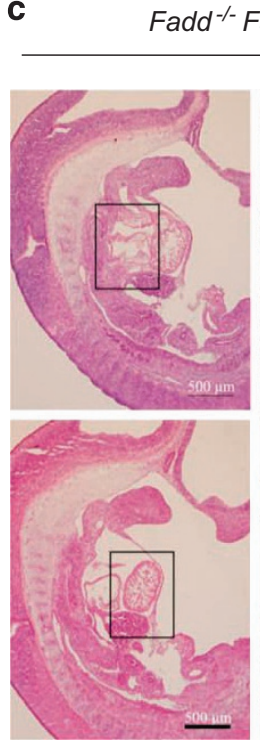

Fadd $^{-/}$Fadd:gfp ${ }^{+}$

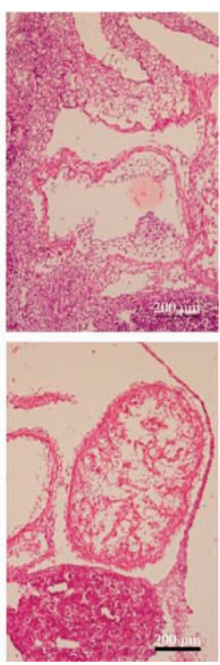

Fadd ${ }^{-/}$Fadd:gfp ${ }^{+}$Tie2-Cre ${ }^{+}$
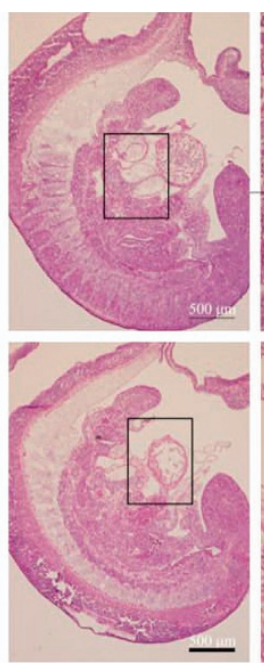
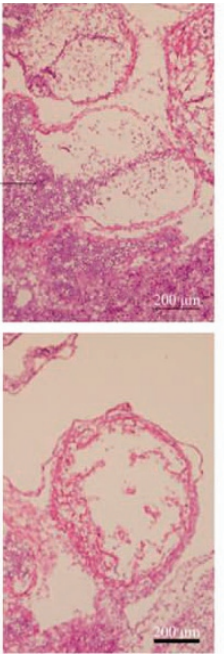

Fadd $^{-/-}$Fadd:gfp ${ }^{+}$Tie2-Cre ${ }^{+}$Ripk3--
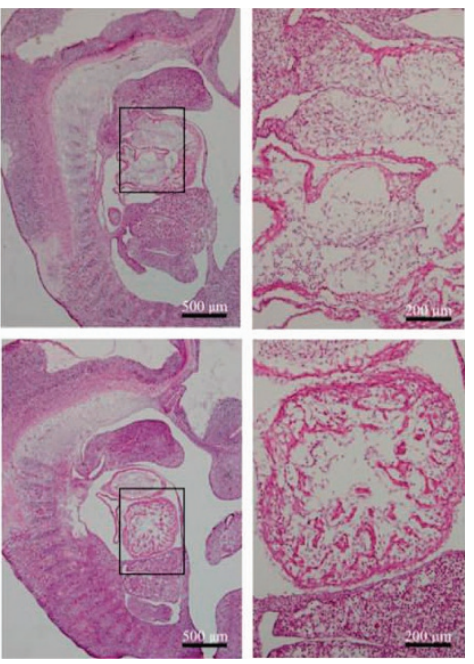

Figure 1 Continued

demonstrated that RIPK3-mediated signaling in Tie-2 expressing cells was responsible for the embryonic lethality of Fadd $^{\prime-}$ with cardiac failure. Further, mechanistic study of cell death in these cell populations will be important for understanding the function of cell death during embryonic development.

\section{Conflict of Interest}

The authors declare no conflict of interest.

Acknowledgements. We thank Dr Jianke Zhang (Thomas Jefferson University, Philadelphia, PA, USA) for providing Fadd ${ }^{-1} \mathrm{Fadd}_{\mathrm{gfp}}{ }^{+}$mice. This work was supported by grants from the Ministry of Science and Technology of China (2016YFSF110034) and the National Natural Science Foundation of China (31571426, 91339104). HZ was supported by Thousand Young Talents Program of the Chinese government.

\footnotetext{
1. Peter ME, Krammer PH. Cell Death Differ 2003; 10: 26-35.

2. Dillon CP et al. Cell Mol Life Sci 2016; 73: 2125-2136.
}

3. Kang TB et al. J Immunol 2004; 173: 2976-2984.

4. Zhang $Y$ et al. J Immunol 2005; 175: 3033-3044.

5. Jiao K et al. Genes Dev 2003; 17: 2362-2367.

6. Moses KA et al. Genesis 2001; 31: 176-180.

7. Yeh WC et al. Science 1998; 279: 1954-1958.

8. Zhang J et al. Nature 1998; 392: 296-300.

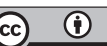

Cell Death and Disease is an open-access journal published by Nature Publishing Group. This work is licensed under a Creative Commons Attribution 4.0 International License. The images or other third party material in this article are included in the article's Creative Commons license, unless indicated otherwise in the credit line; if the material is not included under the Creative Commons license, users will need to obtain permission from the license holder to reproduce the material. To view a copy of this license, visit http://creativecommons.org/licenses/by/4.0/

(C) The Author(s) 2016 